\title{
In Vitro Biocompatibility and Osteoblast Differentiation of an Injectable Chitosan/Nano-Hydroxyapatite/Collagen Scaffold
}

\author{
Yan Chen, ${ }^{1}$ Zhi Huang, ${ }^{2}$ Xiaoming Li, ${ }^{3}$ Songjian Li, ${ }^{4}$ Zhilai Zhou, ${ }^{4}$ Yichen Zhang, \\ Qing ling Feng, ${ }^{5}$ and Bo $\mathrm{Yu}^{4}$
}

${ }^{1}$ Department of Ultrasonic Diagnosis, Zhujiang Hospital of Southern Medical University, Guangzhou 510282, China
${ }^{2}$ School of Geosciences and Info-Physics, Central South University, Changsha 410083, China
${ }^{3}$ Key Laboratory for Biomechanics and Mechanobiology of Ministry of Education, School of Biological Science and Medical Engineering,
Beihang University, Beijing 100191, China
${ }^{4}$ Department of Orthopedics, Zhujiang Hospital of Southern Medical University, Guangzhou 510282, China
${ }^{5}$ State Key Laboratory of New Ceramic and Fine Processing, Tsinghua University, Beijing 100084, China

Correspondence should be addressed to Xiaoming Li, x.m.li@hotmail.com and Bo Yu, gzyubo@gmail.com

Received 28 February 2012; Accepted 3 April 2012

Academic Editor: Shuming Zhang

Copyright ( $) 2012$ Yan Chen et al. This is an open access article distributed under the Creative Commons Attribution License, which permits unrestricted use, distribution, and reproduction in any medium, provided the original work is properly cited.

\begin{abstract}
The purpose of this study was to evaluate the in vitro cell biocompatibility of an in situ forming composite consisting of chitosan (CS), nano-hydroxyapatite and collagen (nHAC), which has a complex hierarchical structure similar to natural bone. MC3T3E1 mouse calvarial preosteoblasts were cultured on the surface of the injectable CS/nHAC and CS scaffold. The proliferations of seeded MC3T3-E1 were investigated for 10 days. Cytotoxicity, cell proliferation, and cell expression of osteogenic markers such as alkaline phosphatase (ALP), type 1 collagen (COL-1), RUNX-2, and osteocalcin (OCN) were examined by biochemical assay and reverse transcription polymerase chain reaction. Cell viability and total cellularity (measured by dsDNA) were similar between the two scaffold groups. However, ALP, COL-1, OCN, and RUNX-2 production were significantly greater when osteoblasts were cultured on CS/nHAC scaffolds. The increase in osteogenic markers production on CS/nHAC scaffolds indicated that these scaffolds were superior to chitosan-only scaffolds in facilitating osteoblast mineralization. These results demonstrate the potential of the CS/nHAC scaffolds to be used in bone tissue engineering.
\end{abstract}

\section{Introduction}

Annually, more than 2.2 million bone grafting procedures (autologous bone graft and banked bone) are performed worldwide to ensure adequate bone healing in many skeletal problems, such as nonunion fractures, cervical and lumbar spine fusion, joint arthrodesis, and revision arthroplasty [1]. Tissue engineering offers a strategy to circumvent those problems. The concept involves the use of a porous and biodegradable scaffold, allowing cells to adhere and proliferate, creating conditions for the formation of ECMlike structures [2-4]. Previous studies have shown that natural-based polymers such as chitosan [5-10] have great potential for bone tissue engineering applications. The main advantages of these materials include low immunogenic potential, bioactive behavior, good interaction with host tissues, chemical versatility, and high availability in nature. Chitosan is a promising material for bone regeneration because it is biocompatible and biodegradable with a degradation rate that is dependent on factors such as degree of deacetylation (DDA) and crystallinity, and it can be easily formed into beads, fibers, or more complex structures $[5,11$, 12].

Bone tissues are mainly constructed from nanosized hydroxyapatite (HA) minerals and type 1 collagen (Col1) matrix with complex hierarchically assembled structures [13-16]. The ideal scaffolds for bone regeneration should promote early mineralization and support new bone formation [17-19]. Mineralized collagen fibrils (nHAC) composites seem to be very promising biomaterials for bone regeneration [20-23]. In the previouse study, the feasibility of developing a thermosensitive and injectable chitosan 
solution in the presence of nHAC was demonstrated [2325]. Combining $\mathrm{nHAC}$ and chitosan has the potential to maximize the beneficial properties of each and creates an injectable scaffold with properties similar to physiological bone which would undoubtedly aid in the formation of new bone at the tissue/biomaterial interface [24-27].

In the present study, the cytocompatibility of the injectable CS/nHAC scaffolds to act as a bone substitude and its potential for bone tissue engineering were investigated in vitro. The ability of the injectable CS/nHAC scaffolds to maintain the viability and functionality of seeded MC3T3-E1 preosteoblasts was investigated in terms of their proliferation and osteoblastic differentiation over 10 days of culture.

\section{Materials and Methods}

2.1. Scaffold Processing. The CS/nHAC composite was prepared by the procedure reported previously [24-27]. nHAC powder was synthesized by self-assembly of nanofibrils of mineralized collagen and sterilized by $\gamma$-ray irradiation $(1.5$ Mrad). Chitosan ( $2 \mathrm{~g}$ ) was dissolved in hydrochloric acid solution $(98 \mathrm{~mL}, 0.1 \mathrm{M})$. The $\mathrm{nHAC}$ powder was added to the chitosan solution $(0.02 \mathrm{~g} / \mathrm{mL})$. Finally, the $\mathrm{pH}$ of the $\mathrm{CS} / \mathrm{nHAC}$ solution was adjusted to 7.0 by adding droplets of $\beta$-glycerophosphate solution $(30 \%(\mathrm{w} / \mathrm{v}))$. The samples for biological tests were prepared in line with the aseptic technique in an aseptic manipulation cabinet. Solutions were injected into a circular mold $(12 \mathrm{~mm}$ diameter and $2 \mathrm{~mm}$ thickness) and solidified in an incubator at $37^{\circ} \mathrm{C}$ for $10 \mathrm{~min}$ to form a hydrogel scaffold.

The CS was prepared similar to the procedure of CS/nHAC. Chitosan ( $2 \mathrm{~g}$ ) was dissolved in hydrochloric acid solution $(98 \mathrm{~mL}, 0.1 \mathrm{M})$, and then the $\mathrm{pH}$ of the CS solution was adjusted to 7.0 by adding droplets of $\beta$-glycerophosphate solution $(30 \%(\mathrm{w} / \mathrm{v}))$. The samples for biological tests were prepared in line with the aseptic technique in an aseptic manipulation cabinet.

2.2. Cell Culture Studies. MC3T3-E1 murine calvarial osteoblasts (subclone 14) cells were purchased from the cell bank of the Chinese Academy of Sciences (Shanghai, China). The cells were grown in a culture medium consisting of $\alpha$-MEM (Sigma, St. Louis, MO), 10\% fetal bovine serum (Biochrom AG, Germany), and 1\% of antibioticantimycotic mixture (Sigma, St. Louis, MO) at $37.8^{\circ} \mathrm{C}$ in a humidified atmosphere of $5 \% \mathrm{CO}_{2}$. When an adequate cell number was obtained, cells at passage 2 were detached with trypsin/EDTA. Cells were seeded at a density of $2 \times$ $10^{4}$ cells/scaffold under static conditions, by means of a cell suspension. The culture medium was changed every 2-3 days until the end of the experiment.

2.3. In Vitro Cytotoxicity Tests. A Cell Count Kit-8 (Cell Count Kit-8, Beyotime, China) was employed in this experiment to quantitatively evaluate the cytotoxicity of the scaffold. Cell viability was assessed after MC3T3-E1 were inoculated on the samples 24-well disks using the CCK-8 kit after $1,3,7$ and 10 days. CCK-8 reagent $(100 \mu \mathrm{L})$ was added to MC3T3 in $1 \mathrm{~mL}$ medium per well. The plates were incubated at $37^{\circ} \mathrm{C}$ for $4 \mathrm{~h}$ and shaken for $1 \mathrm{~min}$. After this, $200 \mu \mathrm{L}(n=5)$ were transferred to 96-well plates and the optical density (OD) was measured on a microplate reader (BioTek, USA) using an absorbance of $490 \mathrm{~nm}$. All the procedures were replicated 3 times.

2.4. Cell Proliferation by DNA Quantification. MC3T3 proliferation on the CS/nHAC scaffolds was determined using a fluorimetric dsDNA quantification kit (PicoGreen, Molecular Probes, Invitrogen, USA). Samples collected at days 1, 3,7 , and 10 were washed twice with a sterile phosphatebuffered saline solution and transferred into $1.5 \mathrm{~mL}$ microtubes containing $1 \mathrm{~mL}$ of ultrapure water. Cell constructs were cryopreserved at $-80^{\circ} \mathrm{C}$ for further analysis. Prior to DNA quantification, samples were thawed and sonicated for $15 \mathrm{~min}$. Standards were prepared with concentrations ranging between 0 and $2 \mathrm{mg} / \mathrm{mL}$. Per each well of an opaque 96-well plate were added $28.7 \mu \mathrm{L}$ of sample $(n=3)$ or standard, $71.3 \mu \mathrm{L}$ of PicoGreen solution, and $100 \mu \mathrm{L}$ of TrisEDTA buffer. The plate was incubated for $10 \mathrm{~min}$ in the dark and fluorescence was measured using an excitation wavelength of $260 \mathrm{~nm}$ and an emission wavelength of $280 \mathrm{~nm}$. All the procedures were replicated 3 times.

2.5. Alkaline Phosphatase Quantification. After culturing for $1,3,7$, and 10 days, the cells were washed and lysed in 0.1 vol\% Triton X-100. The alkaline phosphatase (ALP) activity in the lysis was determined through a p-nitrophenyl phosphate ( $\mathrm{pNPP}$ ) liquid substrate system (Nanjing Jiancheng, China). Five milliliters of each cell lysate solution was added to $195 \mu \mathrm{L}$ of pNPP substrate and incubated in the dark at room temperature for $1 \mathrm{~min}$. The absorbance was read using a plate reader (Molecular Devices, USA) at $405 \mathrm{~nm}$. The intracellular total protein content was determined using a Micro BCA Protein Assay Kit (Nanjing Kaiji, China) and the ALP activity was normalized to it.

\subsection{Osteogenic Differentiation by Reverse Transcriptase PCR.} The expression levels of osteogenesis-related genes were measured using the quantitative reverse transcriptionpolymerase chain reaction (qRT-PCR). The cells were seeded at a density of $2 \times 10^{4}$ cells/well, cultured for $1,3,7$ and 10 days, and then harvested using TRIzol (Gibco) to extract RNA. An equivalent amount of RNA from each sample was reverse-transcribed into complementary DNA (cDNA) using the Superscript II first-strand cDNA synthesis kit (Invitrogen). The qRT-PCR analysis of genes including type 1 collagen (COL-1), osteocalcin (OCN), Runt-related transcription factor 2 (RUNX-2), and $18 \mathrm{~S}$ ribosomal RNA (18 s rRNA) was performed on the Applied Biosystems 7500 using the Quantitect Sybr Green Kit (Qiagen). The primers for the target genes were listed in Table 1 . The Ct values of target genes were normalized by the Ct values of the TaqMan 
TABle 1: Primer sequences and product size for real-time PCR reactions.

\begin{tabular}{lcc}
\hline Target & Sense and anti-sense sequences & bp \\
\hline OCN & 5' AGGGCAATAAGGTAGTGAA & 159 \\
& 5' CGTAGATGCGTCTGTAGGC & \\
RUNX-2 & 5' TGCCCAGTGAGTAACAGAAAGAC & 123 \\
& 5' CTCCTCCCTTCTCAACCTCTAA & \\
COLL & 5' CTTCACCTACAGCACCCTTGT & 120 \\
& 5' AAGGGAGCCACATCGATGAT & \\
18 s rRNA & 5' CCTGGATACCGCAGCTAGGA & 112 \\
& 5' GCGGCGCAATACGAATGCCCC & \\
\hline
\end{tabular}

human housekeeping gene $18 \mathrm{~s}$ rRNA to obtain the DCt values. These values were then subtracted by the $\mathrm{Ct}$ value of the cells cultured on the blank disks to obtain the DDCt values. The fold of change was obtained with $n=3$.

2.7. Statistical Analysis. Statistical significance of differences was determined using one-way and two-way analysis of variance ANOVA. If differences were detected, pairwise comparisons were made using Tukey's HSD test at a confidence interval of $95 \%(P<0.05)$.

\section{Result and Discussion}

3.1. In Vitro Cytotoxicity Tests. The viable cell density increases over initial days up to a plateau based on the CCK-8 assay (Figure 1). The experimental group and control group share similar trend of viable cell density over 10 days. the $\mathrm{CS} / \mathrm{nHAC}$ group reach a maximum value at day 10 , and the CS group reach a maximum value at day 7 ; no significant growth difference was observed between the two groups after 10 days of incubation $(n=5, P>0.05)$. The results of the CCK-8 assay reflect an overall activity which is affected by both cell proliferation and cell viability. The cell proliferation assay showed that cells grew normally with the scaffolds and these scaffolds were nontoxic for MC3T3-E1.

3.2. Cell Proliferation by DNA Quantification. Over the 10 days of the study, the number of cells, as measured by dsDNA, was essentially equivalent between both CS and CS/nHAC scaffold groups (Figure 2). Overall, there was not a significant amount of cell proliferation detected over the course of the study. In vitro cell testing of biomaterials is a well-established method to determine the cytocompatibility of the materials [27-29] by using either cell lines or primary cells. This study was designed primarily to analyze the ability of the composite scaffold to support osteoblast matrix production, and maintaining similar cell numbers in each group allowed differences in these parameters to be studied without the confounding effects of cell number differences. The DNA concentration result of the two groups showed a similar trend to the CCK-8 result. These results demonstrated that both the CS and CS/nHAC scaffold had good cytocompatibility and MC3T3-E1 viability, so it highlights the application of $\mathrm{CS} / \mathrm{nHAC}$ to be a biomaterial for bone tissue engineering.

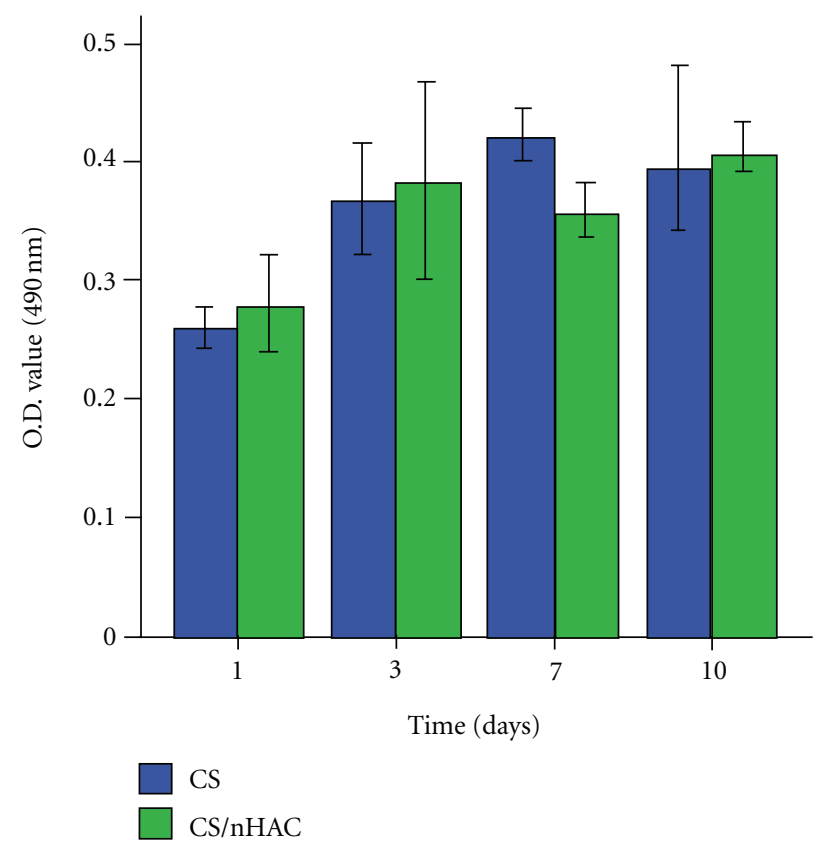

FIGURE 1: CCK-8 assays of MC3T3-E1 proliferation on experimental group and control group after 1, 3, 7, and 10 days of incubation. The values are shown as mean \pm standard deviation $(n=5)$.

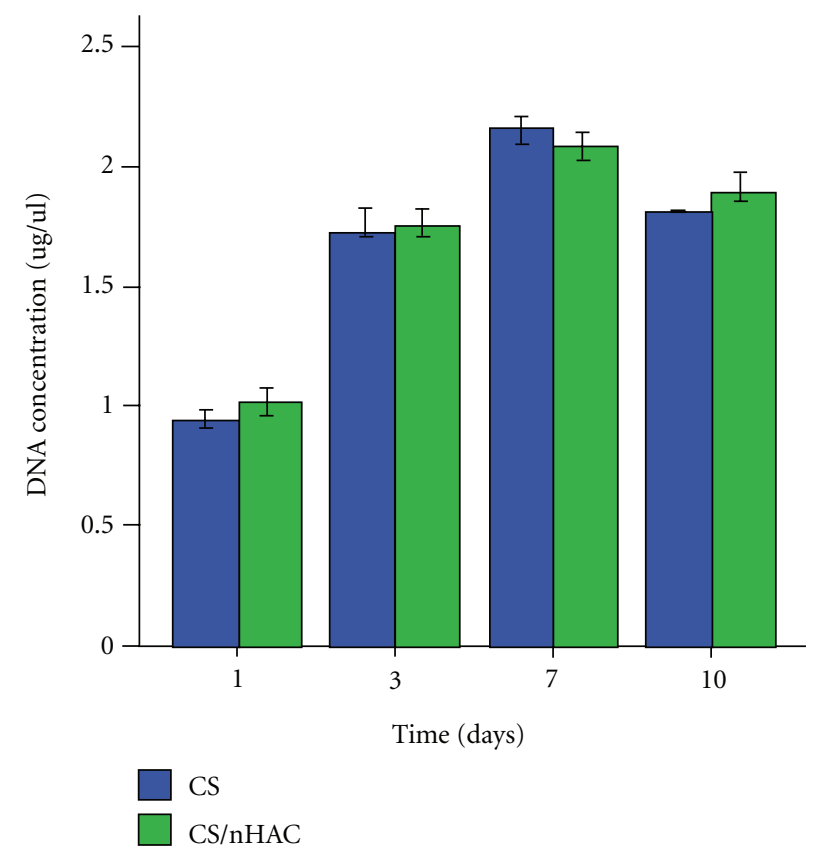

FIGURE 2: DNA concentration of MC3T3-E1 proliferation on experimental group and control group after 1, 3, 7, and 10 days of incubation. The values are shown as mean \pm standard deviation $(n=5)$.

3.3. Alkaline Phosphatase Quantification. ALP activity and calcium mineralization are mostly used as markers for early and late differentiation of osteoblast cells, respectively $[21,22]$. Osteoblastic cell differentiation was assessed by measuring ALP activity normalized to total protein content after $1,3,7$, and 10 days of culture. Figure 3 shows the 


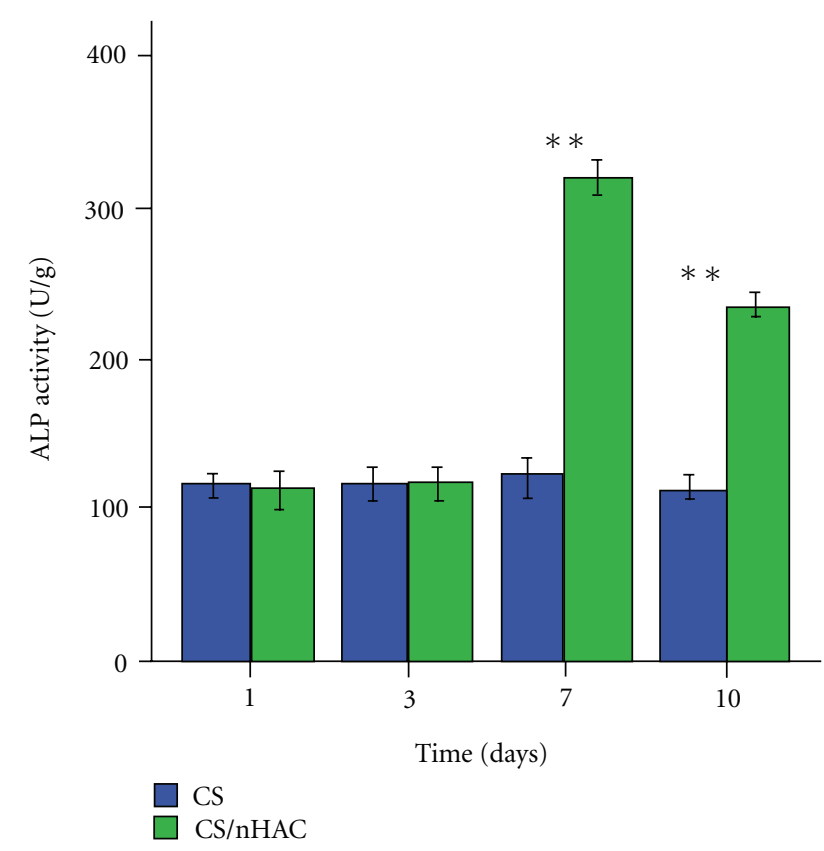

FIgURE 3: ALP assays of MC3T3-E1 proliferation on experimental group and control group after 1,3,7, and 10 days of incubation. The values are shown as mean \pm standard deviation $(n=3)$. Bars with dissimilar letters indicate significantly different values $\left({ }^{* *} P<0.01\right)$.

evolution of ALP activity for MC3T3-E1 cells cultured on the CS/nHAC surfaces in comparison with CS. The ALP activity of MC3T3-E1 cells grown on both groups was not significantly different for day 1 and 3. However, MC3T3-E1 cells cultured on CS/nHAC scaffold had significantly higher ALP activity than those cultured on CS group on day 7 and $10(P<0.001)$. The ALP activity of CS/nHAC group reaching a top on day 7 was observed. The following decrease on day 10 is likely due to reaching an advanced cell culture stage. ALP activity is mostly used as markers for early and late differentiation of osteoblast cells, respectively [30, 31]. These results indicate that the CS/nHAC scaffold can stimulate matrix formation and enhance osteoblast cell differentiation.

3.4. Osteogenic Differentiation by Reverse Transcriptase PCR. A semiquantitative RT-PCR method was used to assess gene expression of osteoblasts after 1, 3, 7, and 10 days. In order to know whether primary osteoblast differentiation was affected by nano-hydroxyapatite/collegen, we selected COL-1, OCN, and RUNX-2 as markers for osteoblastic differentiation in this study. Among bone matrices, COL-1 is the most abundant protein synthesized by active osteoblasts and conductive to mineral deposition. Osteocalcin is secreted solely by osteoblasts and thought to play a role in the body's metabolic regulation and is proosteoblastic or bone-building [28]. It is also involved in bone mineralization and calcium ion homeostasis. RUNX-2 gene is a member of the RUNX family of transcription factors and encodes a nuclear protein with a Runt DNA-binding domain. The protein can bind DNA both as a monomer and, with more affinity, as a subunit of a heterodimeric complex. This protein has been implicated as a key transcription factor associated with osteoblast differentiation [29]. The results of real-time PCR for mRNA expression of osteocalcin and RUNX-2, both of which are osteoblast markers, are represented as fold increases. As shown in Figures 4(a) and 4(b), there are significant differences in the osteocalcin expression between CS/nHAC and $\mathrm{CS}$ at day 1,7 , and $10(P<0.001)$ (Figure $4(\mathrm{a}))$. The osteocalcin expression was 5.5-fold higher on day 7 and 2.7fold higher on day 10 for the CS/nHAC as opposed to the CS. Also, the RUNX-2 expression was significantly higher by day 7 and 10 for the CS/nHAC as opposed to the CS group. The COL-1 expression was significantly lower by day 1 and 3 for the CS/nHAC as opposed to the CS group. However, significant higher COL-1 expression of CS/nHAC than CS was observed at day 7 and $10(P<0.01)$.

Due to the current results in vitro, better osteoblasts viability, and differentiation marked by higher OCN, RUNX2 and COL-1 expression were observed on CS/nHAC surfaces. The addition of nHAC to the chitosan did lead to significantly enhanced osteoblasts differentiation. There are several factors that could contribute to the enhanced mineralized matrix production seen when osteoblasts were cultured on CS/nHAC scaffolds. When calcium phosphatecontaining materials are hydrated in cell culture media, a continuous process of calcium and phosphate ion dissolution and reprecipitation occurs. This creates a dense carbonated apatite layer on the surface of the scaffold that is similar to the structure of native bone and may therefore induce more rapid osteoblast differentiation and mineralization [3032]. Taken together, these gene expression data associated with the osteoblast phenotype provides evidence that the scaffold constitutes a good substrate for MC3T3-E1 cell differentiation leading to ECM mineralization.

\section{Conclusions}

In this study, we conclude that the CS/nHAC scaffold has a suitable biocompatibility for its use as cell culture scaffold for hard tissue regeneration. In vitro experiments revealed more prominent activation of osteoblast differentiation in cells grown on CS/nHAC than in those grown on CS. Collectively, these data indicates the CS/nHAC may give greater results concerning cell proliferation and differentiation compared to chitosan in this in vitro study. We suggest an excellent applicability of the CS/nHAC as bone substitutes.

\section{Authors' Contribution}

Y. Chen and Z. Huang contributed equally to this paper.

\section{Acknowledgments}

The authors are grateful for the financial support from National Natural Science Foundation of China (no. 81101348 and no. 31000431), Natural Science Foundation 


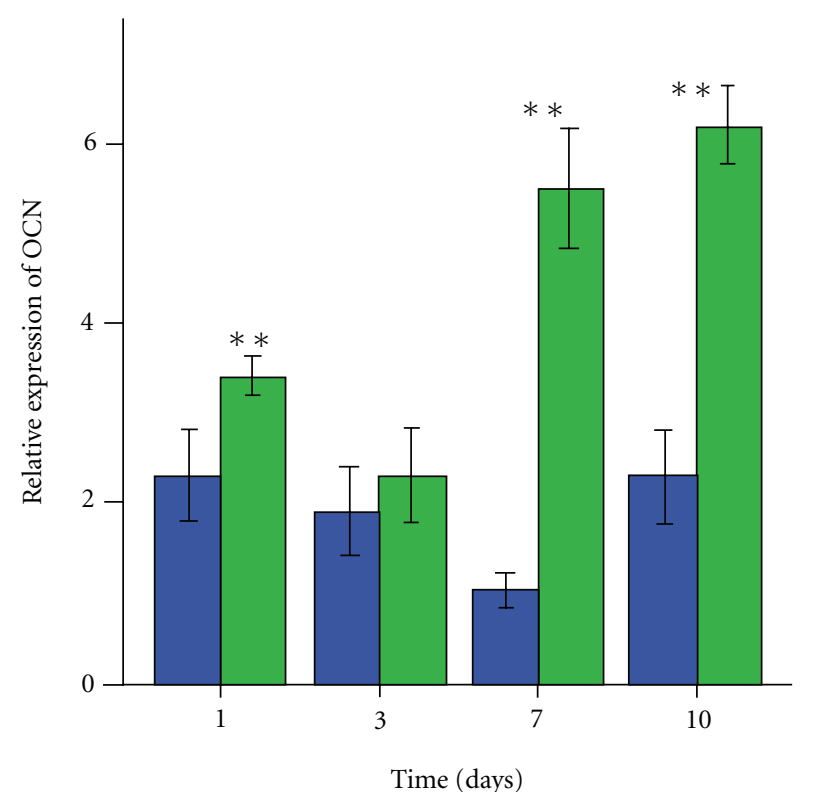

CS CS/nHAC

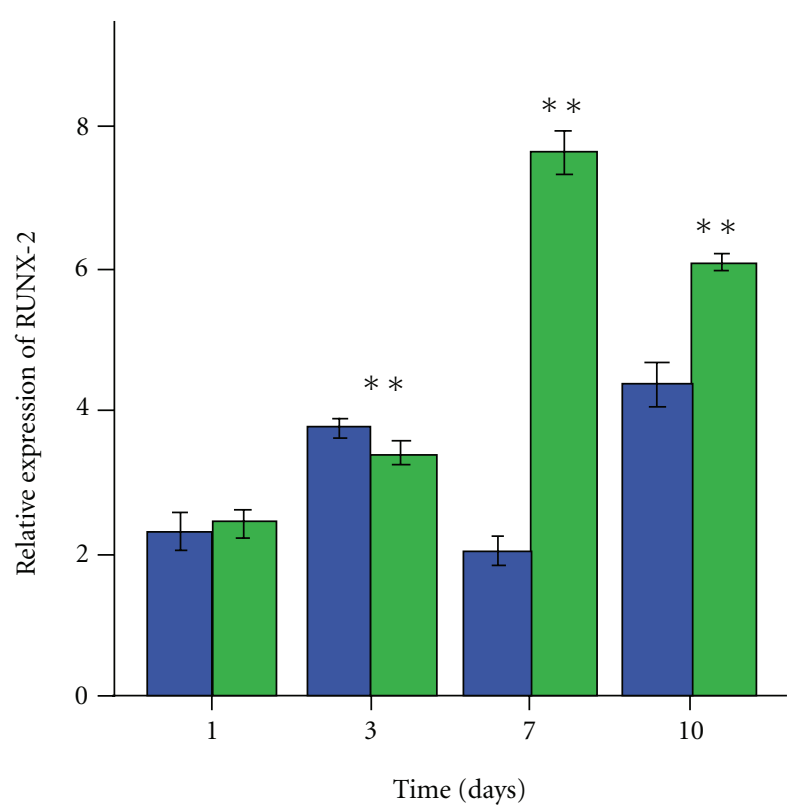

$\square \mathrm{CS}$

(a)

(b)

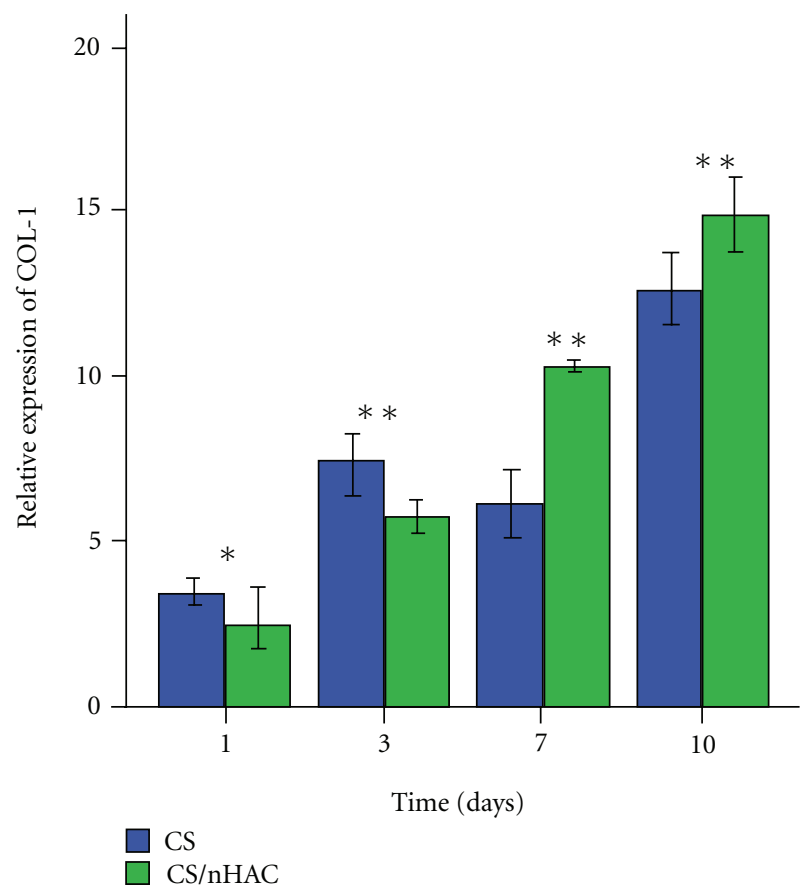

(c)

FIGURE 4: Real-time PCR analysis of osteoblast-specific gene expression. Values were normalized to $18 \mathrm{~s}$ rRNA expression and shown relative to gene expression on (b) for comparison. (a) OCN, (b) RUNX-2, (c) COL-1. The values are shown as mean \pm standard deviation ( $n=3$ ). Bars with dissimilar letters indicate significantly different values $\left({ }^{*} P<0.05,{ }^{* *} P<0.01\right)$.

of Guangdong Province, China (10451051501004727), Guangdong Province Science and Technology project, China (ultrasound evaluation of injectable nanobiomaterials), Doctoral Fund of Ministry of Education,
China (20114433120004), the Beijing Nova Program (no. 2010B011), and State Key Laboratory of New Ceramic and Fine Processing, Tsinghua University, outstanding person fund of Zhujiang Hospital. 


\section{References}

[1] P. V. Giannoudis, H. Dinopoulos, and E. Tsiridis, "Bone substitutes: an update," Injury, supplement 36, pp. S20-S27, 2005.

[2] R. Langer and J. P. Vacanti, “Tissue engineering," Science, vol. 260, no. 5110, pp. 920-926, 1993.

[3] R. Cancedda, B. Dozin, P. Giannoni, and R. Quarto, "Tissue engineering and cell therapy of cartilage and bone," Matrix Biology, vol. 22, no. 1, pp. 81-91, 2003.

[4] D. W. Hutmacher, J. T. Schantz, C. X. Lam, K. C. Tan, and T. C. Lim, "State of the art and future directions of scaffold-based bone engineering from a biomaterials perspective," Journal of Tissue Engineering and Regenerative Medicine, vol. 1, no. 4, pp. 245-260, 2007.

[5] A. Di Martino, M. Sittinger, and M. V. Risbud, "Chitosan: a versatile biopolymer for orthopaedic tissue-engineering," Biomaterials, vol. 26, no. 30, pp. 5983-5990, 2005.

[6] S. H. Elder, D. L. Nettles, and J. D. Bumgardner, "Synthesis and characterization of chitosan scaffolds for cartilage-tissue engineering," Methods in Molecular Biology, vol. 238, pp. 4148, 2004.

[7] Z. Li, H. R. Ramay, K. D. Hauch, D. Xiao, and M. Zhang, "Chitosan-alginate hybrid scaffolds for bone tissue engineering," Biomaterials, vol. 26, no. 18, pp. 3919-3928, 2005.

[8] D. P. Mukherjee, A. S. Tunkle, R. A. Roberts, A. Clavenna, S. Rogers, and D. Smith, "An animal evaluation of a paste of chitosan glutamate and hydroxyapatite as a synthetic bone graft material," Journal of Biomedical Materials Research, vol. 67, no. 1, pp. 603-609, 2003.

[9] L. Kong, Y. Gao, W. Cao, Y. Gong, N. Zhao, and X. Zhang, "Preparation and characterization of nanohydroxyapatite/chitosan composite scaffolds," Journal of Biomedical Materials Research, vol. 75, no. 2, pp. 275-282, 2005.

[10] B. M. Chesnutt, A. M. Viano, Y. Yuan et al., "Design and characterization of a novel chitosan/nanocrystalline calcium phosphate composite scaffold for bone regeneration," Journal of Biomedical Materials Research, vol. 88, no. 2, pp. 491-502, 2009.

[11] L. Qi, Z. Xu, X. Jiang, C. Hu, and X. Zou, "Preparation and antibacterial activity of chitosan nanoparticles," Carbohydrate Research, vol. 339, no. 16, pp. 2693-2700, 2004.

[12] A. K. Azad, N. Sermsintham, S. Chandrkrachang, and W. F. Stevens, "Chitosan membrane as a wound-healing dressing: characterization and clinical application," Journal of Biomedical Materials Research, vol. 69, no. 2, pp. 216-222, 2004.

[13] X. Li, C. A. van Blitterswijk, Q. Feng, F. Cui, and F. Watari, "The effect of calcium phosphate microstructure on bonerelated cells in vitro," Biomaterials, vol. 29, no. 23, pp. 33063316, 2008.

[14] X. Li, H. Liu, X. Niu et al., "Osteogenic differentiation of human adipose-derived stem cells induced by osteoinductive calcium phosphate ceramics," Journal of Biomedical Materials Research, vol. 97, no. 1, pp. 10-19, 2011.

[15] X. M. Li, X. H. Liu, M. Uo, Q. L. Feng, F. Z. Cui, and F. Watari, "Investigation on the mechanism of the osteoinduction for calcium phosphate," Bone, vol. 43, pp. 111-112, 2008.

[16] X. Li, Q. Feng, X. Liu, W. Dong, and F. Cui, "Collagen-based implants reinforced by chitin fibres in a goat shank bone defect model," Biomaterials, vol. 27, no. 9, pp. 1917-1923, 2006.

[17] X. Li, H. Gao, M. Uo et al., "Effect of carbon nanotubes on cellular functions in vitro," Journal of Biomedical Materials Research, vol. 91, no. 1, pp. 132-139, 2009.
[18] X. Li, H. Gao, M. Uo et al., "Maturation of osteoblast-like $\mathrm{SaoS}_{2}$ induced by carbon nanotubes," Biomedical Materials, vol. 4, no. 1, Article ID 015005, 2009.

[19] X. Li, Y. Fan, and F. Watari, "Current investigations into carbon nanotubes for biomedical application," Biomedical Materials, vol. 5, no. 2, Article ID 022001, 2010.

[20] X. Li, X. Liu, J. Huang, Y. Fan, and F. Z. Cui, "Biomedical investigation of CNT based coatings," Surface and Coatings Technology, vol. 206, no. 4, pp. 759-766, 2011.

[21] X. Li, Q. Feng, and F. Cui, "In vitro degradation of porous nano-hydroxyapatite/collagen/PLLA scaffold reinforced by chitin fibres," Materials Science and Engineering C, vol. 26, no. 4, pp. 716-720, 2006.

[22] X. M. Li, X. H. Liu, G. P. Zhang et al., "Repairing $25 \mathrm{~mm}$ bone defect using fibres reinforced scaffolds as well as autograft bone," Bone, vol. 43, p. 94, 2008.

[23] X. M. Li, H. F. Liu, X. F. Niu et al., "The use of carbon nanotubes $\mathrm{v}$ to induce osteogenic differentiation of human adipose-derived MSCs in vitro and ectopic bone formation in vivo," Biomaterials. In press.

[24] Z. Huang, J. Tian, B. Yu, Y. Xu, and Q. Feng, "A bonelike nano-hydroxyapatite/collagen loaded injectable scaffold," Biomedical Materials, vol. 4, no. 5, Article ID 055005, 2009.

[25] Z. Huang, Q. Feng, B. Yu, and S. Li, "Biomimetic properties of an injectable chitosan/nano-hydroxyapatite/ collagen composite," Materials Science and Engineering C, vol. 31, no. 3, pp. 683-687, 2011.

[26] Z. Huang, B. Yu, Q. Feng, S. Li, Y. Chen, and L. Luo, "In situ-forming chitosan/nano-hydroxyapatite/collagen gel for the delivery of bone marrow mesenchymal stem cells," Carbohydrate Polymers, vol. 85, no. 1, pp. 261-267, 2011.

[27] Z. Huang, Y. Chen, Q. L. Feng et al., "In vivo bone regeneration with injectable chitosan/hydroxyapatite/collagen composites and mesenchymal stem cells," Frontiers of Materials Science, vol. 5, no. 3, pp. 301-310, 2011.

[28] Y. Wang, S. Zhang, X. Zeng, L. M. Lwin, A. K. Khiam, and M. Qian, "Initial attachment of osteoblastic cells onto solgel derived fluoridated hydroxyapatite coatings," Journal of Biomedical Materials Research, vol. 84, no. 3, pp. 769-776, 2008.

[29] S. Jalota, S. B. Bhaduri, and A. C. Tas, "In vitro testing of calcium phosphate (HA, TCP, and biphasic HA-TCP) whiskers," Journal of Biomedical Materials Research, vol. 78, no. 3, pp. 481-490, 2006.

[30] S. A. Clarke, N. L. Hoskins, G. R. Jordan, S. A. Henderson, and D. R. Marsh, "In vitro testing of Advanced JAX Bone Void Filler System: species differences in the response of bone marrow stromal cells to $\beta$ tri-calcium phosphate and carboxymethylcellulose gel," Journal of Materials Science, vol. 18, no. 12, pp. 2283-2290, 2007.

[31] W. Att, N. Hori, M. Takeuchi et al., "Time-dependent degradation of titanium osteoconductivity: an implication of biological aging of implant materials," Biomaterials, vol. 30, no. 29, pp. 5352-5363, 2009.

[32] W. Att, N. Hori, F. Iwasa, M. Yamada, T. Ueno, and T. Ogawa, "The effect of UV-photofunctionalization on the timerelated bioactivity of titanium and chromium-cobalt alloys," Biomaterials, vol. 30, no. 26, pp. 4268-4276, 2009. 

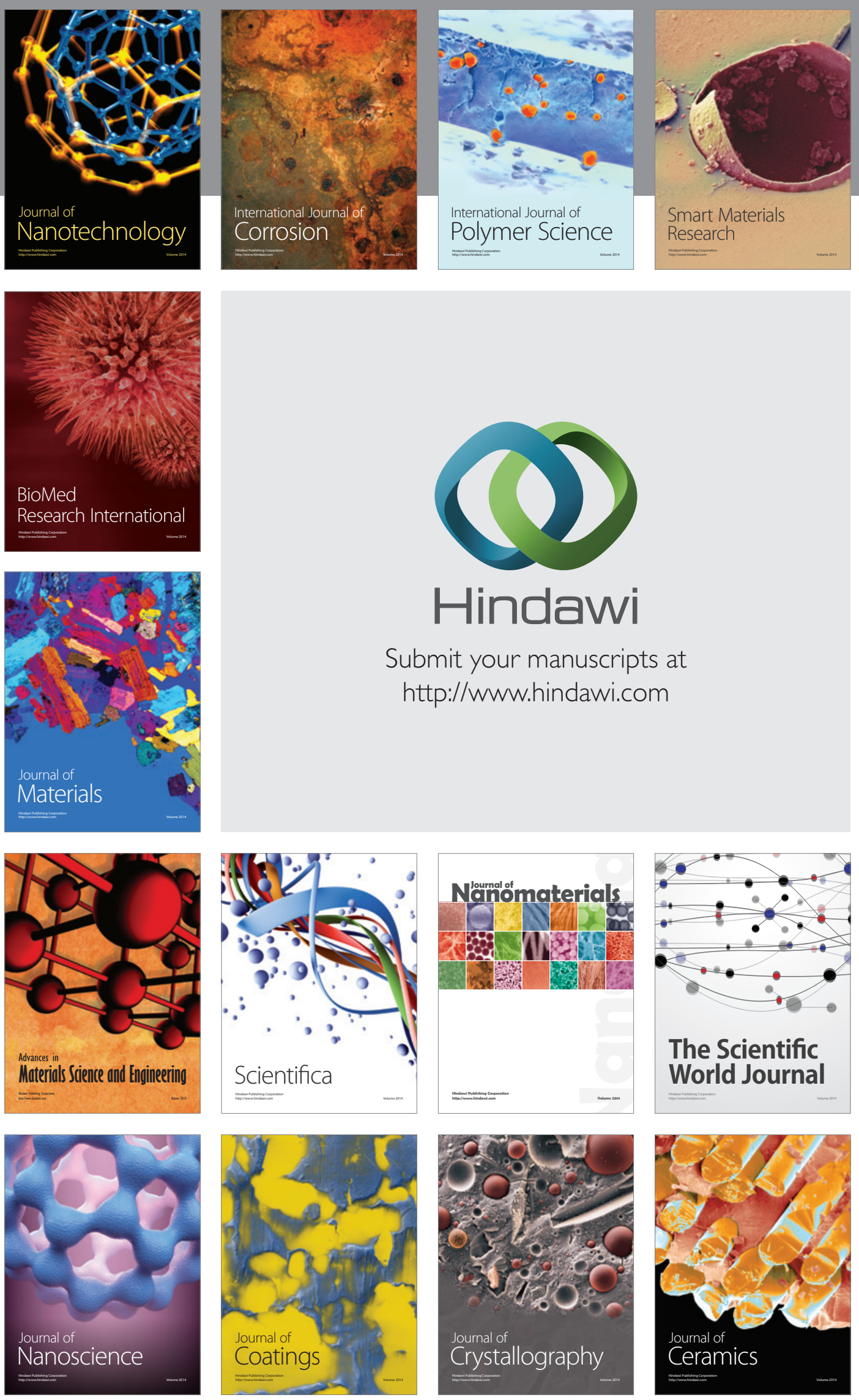

The Scientific World Journal

Submit your manuscripts at

http://www.hindawi.com

\section{World Journal}

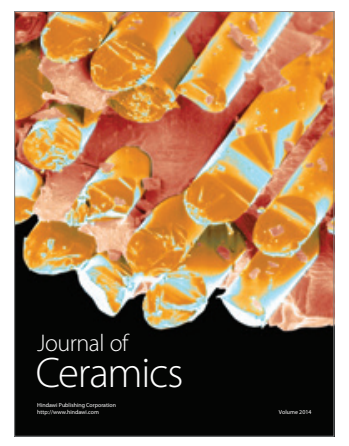

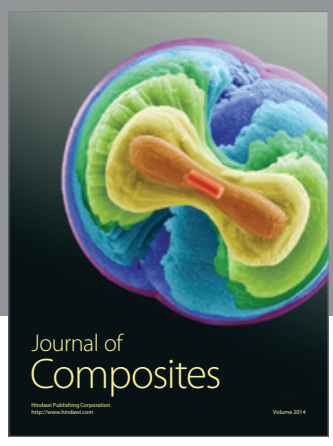
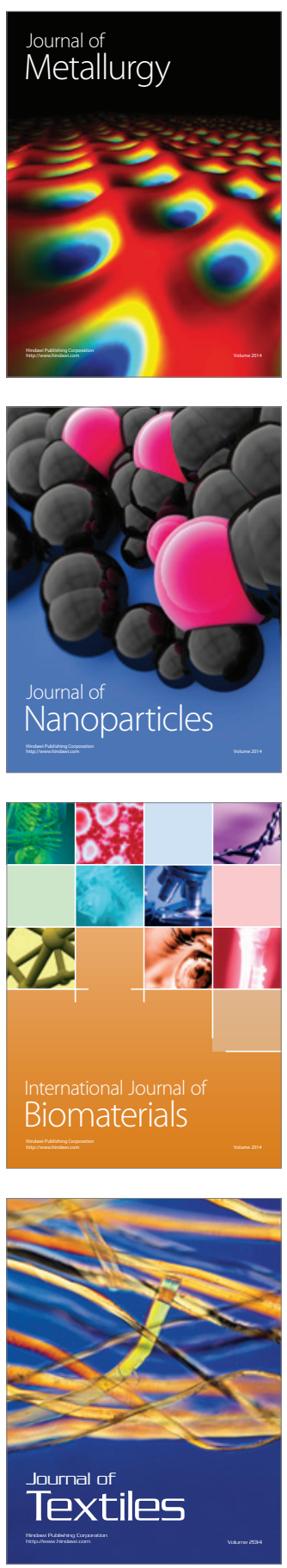